\title{
A perfect plasmonic quarter-wave plate
}

Cite as: Appl. Phys. Lett. 101, 201103 (2012); https://doi.org/10.1063/1.4766189

Submitted: 30 July 2012 . Accepted: 22 October 2012 . Published Online: 13 November 2012

Yuri Gorodetski, Emmanuel Lombard, Aurélien Drezet, Cyriaque Genet, and Thomas W. Ebbesen

ARTICLES YOU MAY BE INTERESTED IN

Sub-wavelength quarter-wave plate based on plasmonic patch antennas

Applied Physics Letters 103, 261108 (2013); https://doi.org/10.1063/1.4858255

Invited Article: Broadband highly efficient dielectric metadevices for polarization control

APL Photonics 1, 030801 (2016); https://doi.org/10.1063/1.4949007

Broadband polarization transformation via enhanced asymmetric transmission through arrays of twisted complementary split-ring resonators

Applied Physics Letters 99, 221907 (2011); https://doi.org/10.1063/1.3664774

\section{Challenge us.}

What are your needs for periodic signal detection?

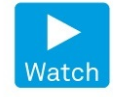

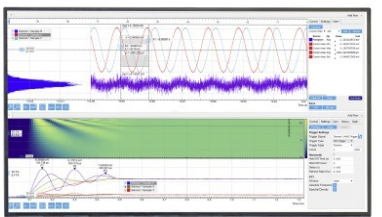

0
Zurich

- Instruments 


\title{
A perfect plasmonic quarter-wave plate
}

\author{
Yuri Gorodetski, ${ }^{1}$ Emmanuel Lombard, ${ }^{1}$ Aurélien Drezet, ${ }^{2}$ Cyriaque Genet, ${ }^{1}$ \\ and Thomas W. Ebbesen ${ }^{1}$ \\ ${ }^{1}$ ISIS and icFRC, Université de Strasbourg and CNRS (UMR 7006), 8, allée Gaspard Monge, \\ 67000 Strasbourg, France \\ ${ }^{2}$ Institut Néel, UPR 2940, CNRS and Université Joseph Fourier, 25, rue des Martyrs, 38042 Grenoble, France
}

(Received 30 July 2012; accepted 22 October 2012; published online 13 November 2012)

\begin{abstract}
The excitation of surface plasmons on an elliptical grating followed by the transmission through a subwavelength aperture can modify the polarization state of the incoming light. The combined effect of the elliptical grooves and a slightly elliptical central hole allows to fully control the birefringence and retardation of the structure, providing a simple approach for polarization state design by the geometry of the structure. From this combination, a perfect plasmonic quarter-wave plate is obtained. (C) 2012 American Institute of Physics. [http://dx.doi.org/10.1063/1.4766189]
\end{abstract}

Through their unique dispersion relations, surface plasmon (SP) modes have provided new ways to modify and tailor light properties at the nanoscale. Two-dimensional confinement of SP modes to the metal surface implies, in particular, that surface corrugations can play a crucial role in the definition of the optical beam properties of the nanostructure, both in the near and the far fields. ${ }^{1,2}$ In this context, the specific polarization constrains of SP modes are attracting increasing attention in order to develop new nanostructured devices for controlling the polarization state of light in subwavelength volumes. Plasmonic elliptical bull's eyes (BE), ${ }^{3,4}$ plasmonic antennas, ${ }^{5,6}$ subwavelength retarders, ${ }^{7,8}$ or very recently uniform aperture plasmonic arrays ${ }^{9}$ and metasurfaces ${ }^{10}$ have been shown to display quarter-wave plate (QWP)-like behaviours. More complex SP based polarization phenomena have also been analyzed in relation with optical chirality, ${ }^{11,12}$ optical spin-Hall effect, ${ }^{13,14}$ and plasmonic Aharonov-Bohm effect. ${ }^{15}$ Polarization control is obviously linked to the ability to induce linear birefringence. Among the different strategies proposed recently, the elliptical BE structure, consisting of a single subwavelength hole surrounded by periodic non-transmissive elliptical corrugations, has the advantage to be easily optimizable. ${ }^{3,16}$

Our previous work ${ }^{3}$ demonstrated that an idealized picture of a phase shift solely associated with the optical path difference between the SP modes propagating along the long and short axes of the ellipse is not enough to reach the optimal values of a perfect quarter-wave plate because of the combined mechanisms of SP propagation over the entire structure and SP transmission through the central hole. In this letter, we demonstrate that minute deformations of the central hole geometry at the nanometer scale give additional phase retardation that are critical in the design of a genuine plasmonic wave plate. We perform a careful study of the polarization properties of a single subwavelength hole together with a full analysis of the cavity-like dynamics that arise in the elliptical corral surrounding the hole. This enables the realization of a nearly-perfect quarter-wave plate.

Because of the BE's ellipticity $\varepsilon=a_{y}-a_{x}$ defined by the radii, $a_{x, y}$, of the first groove along $x$ and $y$ axes, an elliptical BE has a rectangular point symmetry- Fig. 1(a). This symmetry imposes that the Jones matrix, $\boldsymbol{J}$, mapping the incident electric field $\left(E_{x}^{i n}, E_{y}^{i n}\right)^{T}$ to the emerging electric field $\left(E_{x}^{\text {out }}, E_{y}^{\text {out }}\right)^{T}$ transmitted through the structure is diagonal in the $(x, y)$ basis with

$$
\boldsymbol{J} \propto\left(\begin{array}{cc}
1 & 0 \\
0 & \beta
\end{array}\right)
$$

where $\beta=\rho \exp (i \Phi)$ is a complex number. For a $(1,1)^{T}$ incident polarization, $\rho$ corresponds to the relative absorption and $\Phi$ to the retardation phase between the $x$ and $y$ polarization components which measure respectively the relative birefringence and dichroism of the structure.

From symmetry considerations, the addition of a perfect circular hole to the elliptical groove structure cannot by itself introduce any difference between transmitted polarization components. However, it is important to realize that a nanometric deformation of the hole can easily induce polarization asymmetries. A polarization analysis, ${ }^{17}$ performed on single subwavelength holes reveals that although the milling parameters of our focused ion beam (FIB) were set to generate cylindrical holes, deviations from unitary Jones matrices are systematically observed. This turned out to stem from an actually asymmetric milling process. Remarkably, the specific $(x, y)$ raster-scan milling process of the FIB generates deformed holes which have strict rectangular point
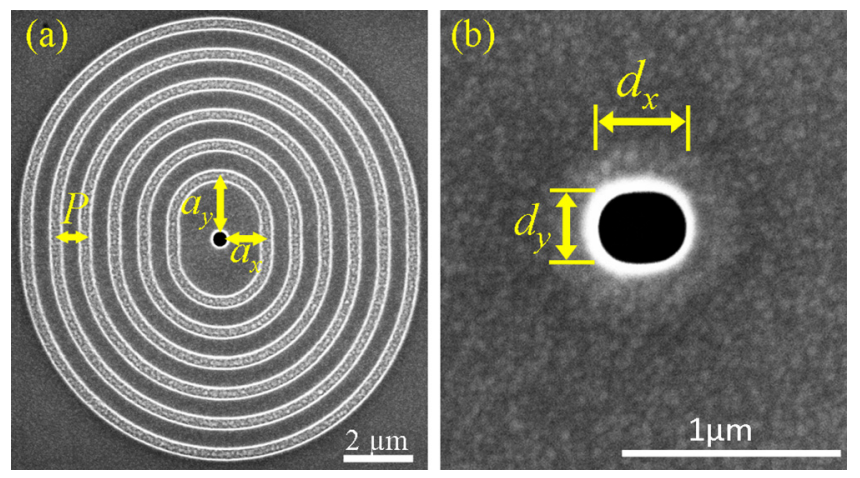

FIG. 1. (a) Elliptical array of non-transmissive grooves of a BE structure for polarization control with periodicity $P=760 \mathrm{~nm}$ and first groove radii $a_{x}=1.79 \times P$ and $a_{y}=1.84 \times P$. (b) Zoom on the central hole with unequal dimensions $d_{x}=300 \mathrm{~nm}$ and $d_{y}=400 \mathrm{~nm}$. The grooves were milled to the depth of $80 \mathrm{~nm}$. 
symmetry, as observed from their diagonal Jones matrices in the $(x, y)$ basis. In order to analyze the birefringence and dichroism induced by the hole, we measure a retardation phase, $\Phi$ and a polarization extinction, $\rho$ in a series of single holes with varying ellipticity, $\Delta_{T}=\left(d_{y}-d_{x}\right) / d$ ( $d=350 \mathrm{~nm}$-nominal hole diameter) milled through $300 \mathrm{~nm}$ thick gold film (see Fig. 1(b)). This study, summarized in Fig. 2(a), shows interestingly that simultaneously null birefringence and dichroism $(\Phi=0, \rho=1)$ are obtained for a non-zero value of $\Delta_{T}$, as a consequence of the FIB error. The results also reveal that very small $\Delta_{T}$ values generate large phase and attenuation changes. The hole shape also influences the spatial distribution of SP modes launched by the edges of the hole. This provides an efficient way to check the geometry of the hole, independently from polarization tomography. To access the SP spatial distribution, we use radiation leakage microscopy $(\mathrm{LRM})^{18}$ of single holes milled through thin $(50 \mathrm{~nm})$ gold film, while varying their ellipticities. Each hole was illuminated by circularly polarized light and the leakage signal was collected through an oilimmersion high numerical aperture objective. Imaging the Fourier plane (k-space) enables us to quantify the influence of the hole geometry by monitoring the leakage intensity distribution along the "plasmonic circle" $-k_{x}^{2}+k_{y}^{2}=k_{S P}$ $\left(k_{S P}=2 \pi / \lambda_{S P}\right)$. The peak-to-dip intensity variation $\left(I_{x}-I_{y}\right)$ as a function of the hole ellipticity (Fig. 2(b)) exhibits a similar behaviour as the data measured by polarization tomography. Remarkably, the correspondence between these two curves not only demonstrates the polarization effect of the

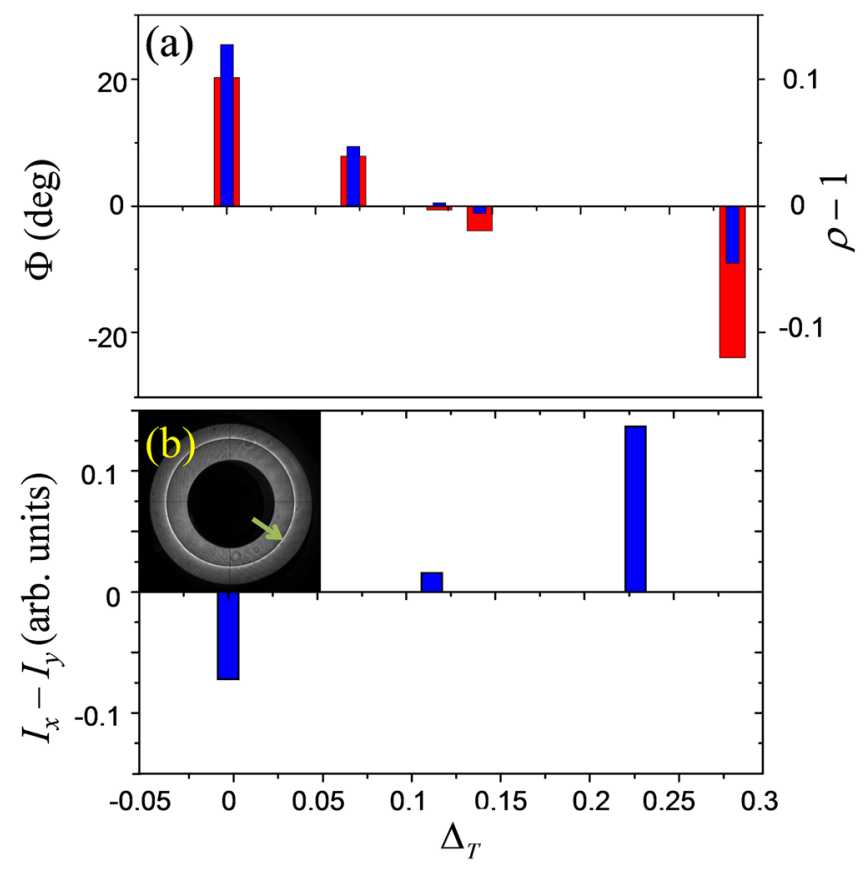

FIG. 2. (a) Retardation phase (red bars) and $\rho-1$ (blue bars) measured as a function of the hole ellipticity. The incident polarization state is prepared using a polarizer, a half-wave plate, and a QWP. The transmitted light (laser at $\lambda=785 \mathrm{~nm}$ ) is analyzed by an additional QWP followed by an analyzer. The full Mueller matrix is measured following the procedure described in Ref. 17. As no depolarization was detected, an equivalent Jones matrix was calculated from which $\rho$ and $\Phi$ were extracted. (b) Intensity modulation of the leakage radiation in the Fourier plane as a function of the hole ellipticity. Inset-a typical intensity distribution, captured in the LRM Fourier plane. The arrow points to the SP wave-vector circle $k_{S P}$. hole deformation, but also enables one to calibrate the fabrication process.

The second main contribution to the definition of the $(\Phi, \rho)$ parameters is the effect on the transmitted light of the constructive interferences of standing SP waves excited inside the cavity formed by the periodic array of elliptical grooves surrounding the hole. To quantify this precisely, we fabricated a series of circular BE structures where the radius of the innermost groove, $a$, was varied between $a=0.9 \times P$ and $a=2.9 \times P$ while keeping the groove periodicity, $P=760 \mathrm{~nm}$, constant and resonant with the incident wavelength. Figure 3(a) shows the evolution of the transmitted intensity as a function of $a / P$ (red circles). The periodic shape of the plot reveals the progressive build-up of constructive phase conditions that correspond to a cavity mode in the structure. ${ }^{19}$ Cavity resonances are associated both with phase and intensity modulations that we elucidate from a simple model which evaluates the field at the hole as a Fano interference between the directly transmitted light and a SP wave launched by the array of grooves as

$$
E_{h}=1+\gamma \sqrt{a} \exp \left(i k_{S P} a\right)+r \gamma \sqrt{a} \exp \left(3 i k_{S P} a\right) .
$$

Here, $E_{h}$ stands for the transmitted field amplitude relative to the one of the single hole. The first term represents the incident field, the second describes the SP field going directly from the plasmonic grating to the central hole, whereas the

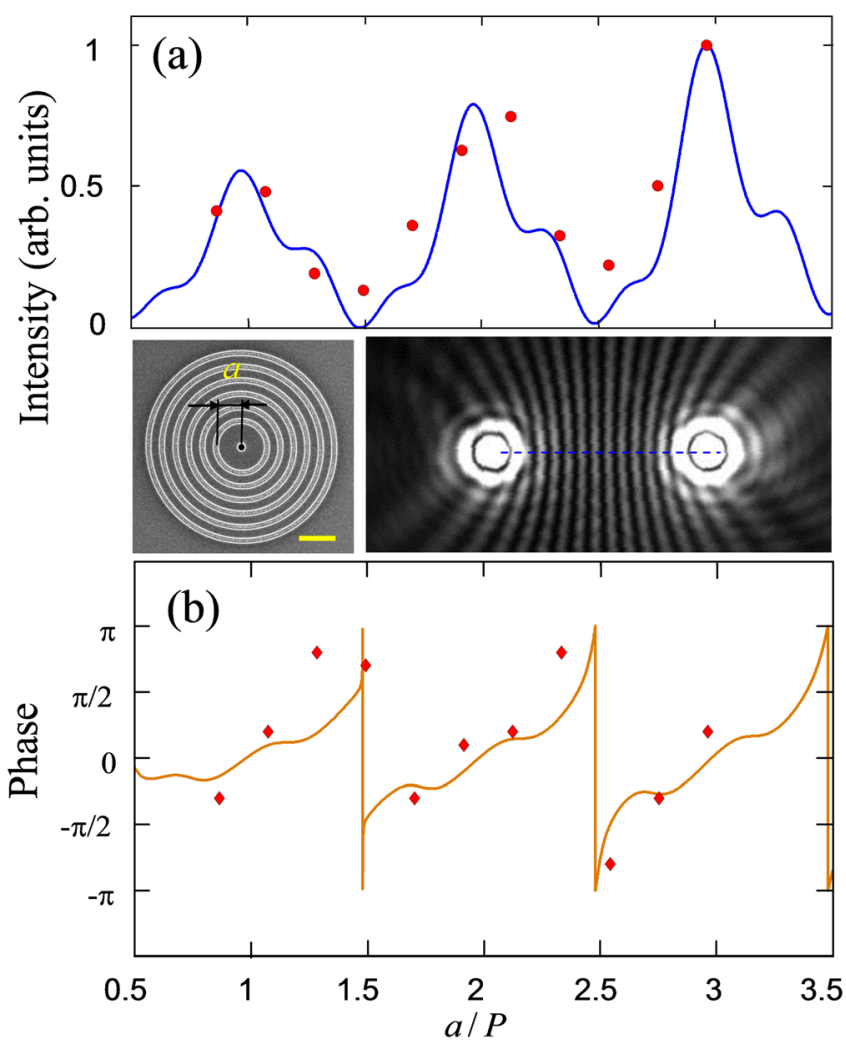

FIG. 3. (a) Normalized intensity transmitted through a circular BE as a function of $a / P$. Red circles are measured values and the solid blue line is the model with a best fitting model parameters $r=0.2+0.2 i$ and $\gamma=0.76$. (b) Phase of the cavity mode as a function of $a / P$. Diamonds are the values extracted from measured interference patterns and the red solid line represents the model. Insets show the geometry of the BE structure and a typical interference pattern of a pair of bull's eyes separated by $15 \mu \mathrm{m}$. The scale bar is $2 \mu \mathrm{m}$. Cross-sections were measured along the blue dashed line. 
third term represents the SP field that goes from one side of the grating to the other one, and then is reflected back to the central hole. The $\gamma$ parameter is the coupling efficiency of the plasmonic grating and $r$ is the SP reflection coefficient from a groove edge. The $\sqrt{a}$ coefficient represents the field focusing as a result of the radial SP propagation. ${ }^{19}$ This model is in a good agreement with our measurements and we obtain the best fit with $r=0.2+0.2 i$ and $\gamma=0.76$ - see Fig. 3 (a) (solid line). A phase of the transmitted field is extracted from this model, as represented in Fig. 3(b) (solid line) as a function of $a / P$. We verify quantitatively this phase dependence by directly measuring it by a simple interference experiment. We fabricate pairs of bull's eyes with unequal $a$ and measure the interference fringe patterns appearing in the far field. The fringe patterns (inset Fig. 3) are crosssectioned along the center line of the hole pairs and their shift with respect to a reference pattern of two identical bull's eyes with $a=0.9 \times P$ is measured (Fig. 3(b) diamonds). It is evident that the phase changes periodically with $a / P$. The qualitative agreement of the measured phase shifts with the model prediction provides a way to estimate and design the phase modulation in the transmission. Importantly, the ability to locally control both phase and intensity of transmitted light is a promising route towards extended field shaping using plasmonic interactions.

The total phase retardation and extinction between the $x$ and $y$ polarization components in the elliptical BE can now be understood from a superposition argument, with the retardation being given by a phase difference between the cavity modes of two circular BEs with dimensions of $a_{x}$ and $a_{y}$. In order to describe solely the $\mathrm{BE}$ grooves contribution, we carefully selected the appropriate $\Delta_{T}=0.11$ value which gives nearly unity Jones matrix, $\boldsymbol{J}_{h} \propto$ $\left[\begin{array}{cc}1 & 0.06 e^{i 0.38 \pi} \\ 0.05 e^{i 0.39 \pi} & 1.02 e^{i 0.018 \pi}\end{array}\right]$. With such a hole at the centre of the elliptical bull's eye, we analyzed, by the tomography method, the associated Jones matrices $\mathbf{J}_{B E}$ as a function of the long and short axes dimensions $a_{x}$ and $a_{y}$. Our aim was to achieve a $\pi / 2$ dephasing between the two polarization components while keeping the same intensity in transmission (i.e., minimizing any dichroic effect). We chose values for the minor and major radii $a_{x}=1.54 \times P$ and $a_{y}=2.04 \times P(\varepsilon=P / 2)$. It is evident from the model that as the phase varies smoothly in this region, the intensity drops from the resonant peak. Accordingly, there is a trade-off between the transmission intensity and phase retardation. Experimentally, the Jones matrix of this structure was found to be

$$
\boldsymbol{J}_{B E} \propto\left[\begin{array}{cc}
1 & 0.03 e^{i 0.51 \pi} \\
0.03 e^{i 0.70 \pi} & 0.87 e^{i 0.41 \pi}
\end{array}\right]
$$

leading to a retardation of $\Phi_{B E}=73.8^{\circ}$ and a dichroism of $\rho_{B E}=0.87$. These values are close to the previously reported ones. ${ }^{3}$ This dichroism is too large to be related to a natural damping of SPs propagating over $a_{x}$ and $a_{y}$. This additional dichroism when accounted for the actual ellipticity of the BE $\varepsilon=P / 2$ falls into the noise level of the experiment $(\sim 3 \%)$, henceforth justifying neglecting imaginary part of $k_{S P}$ for the modeling in Eq. (2).
One of the central targets of this work is to improve the retardation and the dichroism towards the desired values $(\Phi=\pi / 2, \rho=1)$. This can be done by squeezing the central hole of the BE, to an ellipticity value, precisely chosen from the data in Fig. 2. From a simple factorization argument on (a priori diagonal) Jones matrices with $\mathbf{J}_{W P}=\mathbf{J}_{B E} \cdot \mathbf{J}_{h}$, one is seeking a hole-induced retardation of $\pi / 2-\Phi_{B E}$ while compensating a relative absorption $\left|E_{y} / E_{x}\right|=\rho_{B E}$. This compensation will only be possible if the principle axes of the deformed hole are flipped with respect to those of the elliptical BE, so that at the level of the hole, $\left|E_{y} / E_{x}\right|$ turns larger than 1 when expressed in the $(x, y)$ basis.

We find that a central hole milled with $\Delta_{T}=0$ (corresponding to an actual squeezing of $-40 \mathrm{~nm}$ with its long axis aligned along $x$; Fig. 2), displays the following Jones matrix

$$
\boldsymbol{J}_{h} \propto\left[\begin{array}{cc}
1 & 0.036 e^{i 0.41 \pi} \\
0.05 e^{i 0.39 \pi} & 1.12 e^{i 0.09 \pi}
\end{array}\right],
$$

with indeed a compensating dichroic factor larger than one. Finally, the Jones matrix of the elliptical BE combining both this hole and the previously designed elliptical grating is measured as

$$
\boldsymbol{J}_{W P} \propto\left[\begin{array}{cc}
1 & 0.03 e^{i 0.56 \pi} \\
0.03 e^{i 0.70 \pi} & 0.96 e^{i 0.50 \pi}
\end{array}\right],
$$

which is indeed very close to the simple $\boldsymbol{J}_{B E} \cdot \boldsymbol{J}_{h}$ product, giving a full retardation of $\Phi_{W P}=89.9^{\circ}$ and a dichroism of the order of $3 \%$ only, with residual deviations from the perfect quarter-wave plate within experimental errors. Note that the proposed scheme does not rely on specific sizedependent features and thus enables, in the plasmonic regime, the design of quarter-wave plates for different wavelengths. Using the hole ellipticity as a residual dichroism compensating degree of freedom, the small size of the structures along with their resonant transmission are interesting when aiming at producing large area polarizing arrays with wideband spectral responses.

To summarize, a subwavelength hole might be the simplest optical device, it nevertheless displays surprisingly rich polarization properties. ${ }^{12,20,21}$ These properties turn out to be crucial in the combination with the cavity dynamics of an elliptical BE structures for generating almost perfect quarter-wave plates. This demonstrates the relevance of the bull's eye architecture in the design of plasmonic nanodevices. The wide parameter space opened by such composite structures is a promise for many applications in photonics requiring local addressing or ultrafast information storage technology.

${ }^{1}$ W. L. Barnes, A. Dereux, and T. W. Ebbesen, Nature 424, 824 (2003).

${ }^{2}$ A.V. Zayats, I. I. Smolyaninov, and A. A. Maradudin, Phys. Rep. 408, 131 (2005).

${ }^{3}$ A. Drezet, C. Genet, and T. W. Ebbesen, Phys. Rev. Lett. 101, 043902 (2008).

${ }^{4}$ N. Yu, Q. J. Wang, C. Pflügl, L. Diehl, F. Capasso, T. Edamura, S. Furuta, M. Yamanishi, and H. Kan, Appl. Phys. Lett. 94, 151101 (2009).

${ }^{5}$ P. Biagoni, M. Savoini, J.-S. Huang, L. Duo, M. Finazzi, and B. Hecht, Phys. Rev. B 80, 153409 (2009).

${ }^{6}$ A. Pors, M. G. Nielsen, G. Della Valle, M. Willatzen, O. Albrektsen, and S. I. Bozhevolnyi, Opt. Lett. 36, 1626 (2011).

${ }^{7}$ E. H. Khoo, E. P. Li, and K. B. Crozier, Opt. Lett. 36, 2498 (2011). 
${ }^{8}$ P. Chimento, N. V. Kuzmin, J. Bosman, P. F. A. Alkemade, G. W. 't Hooft, and E. R. Eliel, Opt. Express 19, 24219 (2011).

${ }^{9}$ A. Roberts and L. Lin, Opt. Lett. 37, 1820 (2012).

${ }^{10}$ Y. Zhao and A. Alu, Phys. Rev. B 84, 205428 (2011).

${ }^{11}$ A. Drezet, C. Genet, J. Laluet, and T. W. Ebbesen, Opt. Express 16, 12559 (2008).

${ }^{12}$ V. A. Fedotov, P. L. Mladyonov, S. L. Prosvirnin, A. V. Rogacheva, Y. Chen, and N. I. Zheludev, Phys. Rev. Lett. 97, 167401 (2006).

${ }^{13}$ Y. Gorodetski, A. Niv, V. Kleiner, and E. Hasman, Phys. Rev. Lett. 101, 043903 (2008).

${ }^{14}$ Y. Gorodetski, K. Y. Bliokh, B. Stein, C. Genet, N. Shitrit, V. Kleiner, E. Hasman, and T. W. Ebbesen, Phys. Rev. Lett. 109, 013901 (2012).

${ }^{15}$ Y. Gorodetski, S. Nechayev, V. Kleiner, and E. Hasman, Phys. Rev. B 82, 125433 (2010).
${ }^{16}$ O. Mahboub, S. Carretero Palacios, C. Genet, F. J. Garcia-Vidal, S. G. Rodrigo, L. Martin-Moreno, and T. W. Ebbesen, Opt. Express 18, 11292 (2010).

${ }^{17}$ E. Lombard, A. Drezet, C. Genet, and T. W. Ebbesen, New J. Phys. 12, 023027 (2010).

${ }^{18}$ A. Drezet, A. Hohenau, D. Koller, A. Stepanov, H. Ditlbacher, B. Steinberger, F. R. Aussenegg, A. Leitner, and J. R. KrennGordon, Mater. Sci. Eng., B 149, 220 (2008).

${ }^{19}$ S. Carretero-Palacios, O. Mahboub, F. J. Garcia-Vidal, L. Martin-Moreno, S. G. Rodrigo, C. Genet, and T. W. Ebbesen, Opt. Express 19, 10429 (2011).

${ }^{20}$ J.-M. Yi, A. Cuche, F. de León-Pérez, A. Degiron, E. Laux, E. Devaux, C. Genet, J. Alegret, L. Martín-Moreno, and T. W. Ebbesen, Phys. Rev. Lett. 109, 023901 (2012).

${ }^{21}$ R. Gordon, A. G. Brolo, A. McKinnon, A. Rajora, B. Leathem, and K. L. Kavanagh, Phys. Rev. Lett. 92, 037401 (2004). 